\title{
Approches des genres dans l'enseignement apprentissage de la production verbale écrite à l'école élémentaire française
}

\author{
Brigitte Marin \\ CIRCEFT (EA 4384, Universités de Paris 8 et de Paris Est Créteil) et GDR CNRS 2657 \\ brigitte.marin@creteil.iufm.fr \\ Jacques Crinon \\ CIRCEFT (EA 4384, Universités de Paris 8 et de Paris Est Créteil) et GDR CNRS 2657 \\ jacques.crinon@creteil.iufm.fr
}

\begin{abstract}
L'apprentissage des genres est un objectif majeur de l'enseignement apprentissage de la production écrite. Écrire ne consiste pas seulement à savoir former et assembler des phrases ; un texte résulte d'une intention globale en lien avec la pratique sociodiscursive au sein de laquelle sa rédaction se situe. Il emprunte des formes historiquement associées aux pratiques d'une communauté (Bernié, 2002 ; Wenger, 1998) et constamment revivifiées par cette communauté discursive. Pour parler dans les termes de Bakhtine, on pourrait définir un genre de discours comme « un type relativement stable d'énoncés » (du point de vue à la fois des contenus, du style et de la construction du texte) qui correspond à «la spécificité d'une sphère d'échange » (Bakhtine, 1984, p. 265).

L'accent mis sur les genres en didactique du français, depuis bientôt une trentaine d'années (notamment Eva, 1996 ; Garcia-Debanc, 1989 ; Dolz \& Schneuwly, 1998), sur la diversification des textes à produire (Schneuwly, 1990) et sur la prise en compte des caractéristiques particulières de chaque genre est allé de pair avec une tendance à intégrer les activités de production d'écrit aux pratiques didactiques des différentes disciplines scolaires, en France (Baudry, Bessonnat, Laparra \& Tourigny, 1997 ; Jaubert, 2007), comme dans d'autres pays (Britton, 1970). Elle s'articule aussi avec une réflexion d'ensemble sur le rôle de la littéracie étendue (Bautier, 2008) dans les apprentissages et la réussite scolaire des élèves. La maîtrise des genres, affirment même Tardy et Swales (2008), apparaît aujourd'hui comme une forme particulièrement discriminante de capital symbolique.
\end{abstract}

Aussi importe-t-il de mieux comprendre comment les jeunes élèves, dès l'école primaire, apprennent à écrire « dans les genres », c'est-à-dire d'une part comment ils acquièrent une représentation de l'activité d'écriture comme d'une activité «située », "adressée » et régie par des intentions, et d'autre part comment ils s'approprient les connaissances et les savoir-faire relatifs aux genres qu'ils pratiquent en classe, au sein de disciplines différentes.

Nous avons fait écrire à plusieurs reprises des élèves de classes de cours moyen ( $4^{\mathrm{e}}$ et $5^{\mathrm{e}}$ années) dans deux genres contrastés, texte explicatif scientifique et récit de fiction (épisodes de roman d'aventures).

Le but de cette communication est, à partir des corpus ainsi obtenus, de décrire les acquis des élèves et les obstacles (Fabre, 1999) qu'ils rencontrent dans leur apprentissage de la production de textes appartenant à ces genres. Car c'est, pensons-nous, sur de telles observations que peut s'appuyer ultérieurement la construction de démarches d'enseignement qui prennent en compte les élèves. Il s'agira en outre de montrer l'évolution des textes au cours du travail (dans une séquence et sur l'année) et de mettre ces évolutions en relation avec certaines des particularités didactiques des situations proposées.

\section{Genre explicatif et récit d'aventures}

Quelles sont les caractéristiques respectives des deux genres visés dans cette recherche ? Une première distinction concerne les mondes convoqués par les textes. Les contenus transmis par un texte scientifique 
peuvent être jugés vrais ou faux selon leur adéquation à un corpus de savoirs de référence. Un texte de fiction décrit un monde inventé ${ }^{1}$. Opposition qui peut laisser croire aux jeunes scripteurs que, dans la fiction, tout est permis. Il n'en est pourtant rien; le contrat de lecture proposé par le roman d'aventures fait la part belle à la description réaliste d'un monde inconnu ou éloigné où un personnage doit survivre et affronter des dangers. Les règles de vraisemblance propres au genre imposent donc à l'auteur d'utiliser des informations conformes à une expérience partagée du monde ou renvoyant à des connaissances géographiques vérifiables ; elles créent un « effet d'univers sémantique» (Rastier, 2007).

Dès lors, l'univers tendant à rendre compte de la réalité - dans le texte scientifique - et l'univers créé par l'imagination - dans le roman d'aventures - ne s'opposent pas directement, du point de vue de la valeur épistémique des informations qu'on y trouve, mais plutôt par l'étendue des connaissances antérieures qui permettent de les construire. À l'ensemble limité des connaissances attendues pour répondre à une question scientifique s'oppose l'ensemble infini des connaissances au sein desquelles l'auteur d'un roman peut puiser pour donner l'illusion d'un monde.

Une deuxième distinction concerne le type de cohérence causale qui gouverne chacun de ces genres. Dans un genre comme dans l'autre, les relations causales entre les informations jouent un rôle majeur dans l'organisation du texte. Cela apparaît évident pour le texte scientifique explicatif : expliquer, c'est justement faire connaître la raison ou la cause de quelque chose, ou encore, selon Combette et Tomassone (1988), repris par Adam (1997), faire comprendre un phénomène à partir d'une question. Mais c'est vrai aussi du récit, dont la structure chronologique est soutenue par une structure causale. Il s'agit cependant de deux types différents de causalité. Alors que le texte scientifique met en jeu la causalité du monde physique, c'est-à-dire des relations entre des «évènements " (il s'agit par exemple de répondre à la question: «pourquoi le niveau des océans monte-t-il?»), le récit, qui met en scène des personnages, s'organise en fonction de relations entre les états intentionnels de ceux-ci et leurs actions (par exemple : « Pourquoi le personnage se réfugie-t-il dans un arbre ?») (Voir Denhière \& Baudet, 1992).

Une autre différence concerne le degré de généralité des explications. D'un côté il s'agit d'expliquer des événements uniques (par exemple, pourquoi le personnage est perdu dans la forêt), de l'autre on s'intéresse à des évènements récurrents (pourquoi le niveau des océans s'élève) (Ohlsson, 2002). Raconter les aventures d'un personnage demande de détailler les circonstances particulières de l'enchainement de celles-ci. Écrire un texte scientifique nécessite de ne conserver que ce qui a valeur d'explication générale, valable pour toutes les occurrences du phénomène. Les observations ou les expériences qui ont permis d'établir cette explication ne peuvent alors être invoquées qu'en tant que preuves, non pour elles-mêmes. L'énonciateur s'efface, l'inscription dans une temporalité, marquée par le recours à des temps verbaux du passé, également, laissant place au présent général. Le raisonnement scientifique est atemporel et semble s'énoncer de lui-même.

\section{Corpus}

\subsection{Recueil des données}

Les données recueillies dans le cadre de cette recherche constituent un vaste corpus d'écrits, hétérogène du point de vue (i) du genre - textes explicatifs à visée scientifique $v s$. textes de fiction -, (ii) de leur stade d'élaboration - première version d'écriture vs. version révisée - et (iii) de leur statut - textes provoqués par une consigne d'écriture scolaire et destinés à une publication en ligne $v s$. critiques envoyées à des partenaires distants. En effet, lors de chaque séquence de travail ${ }^{2}$, pour un genre de texte donné, les élèves dont nous retenons les textes ici ont assumé une position de tuteurs; ils ont émis par écrit et envoyé par courrier électronique des critiques et conseils destinés à aider des pairs à réviser les textes que ceux-ci avaient écrits. Dans le cadre de cette étude, nous ne prenons en compte que les élèves tuteurs dans la perspective de la construction du genre dans lequel s'inscrit leur activité de scripteur, de critique et de réviseur ${ }^{3}$. 
Le travail a été conduit ${ }^{4}$ dans huit classes de $4^{\mathrm{e}}$ et $5^{\mathrm{e}}$ années de la banlieue parisienne, dont certaines, situées dans des secteurs défavorisés, sont composées d'élèves pour la majorité en difficulté. Nos analyses se fondent sur les productions d'écrit de 144 élèves - sur les 220 élèves qui ont participé à l'expérimentation - constitués en groupes comprenant en nombre égal des élèves de bon niveau et de faible niveau ${ }^{5}$. Cependant, dans le cadre de cette étude, nous avons pris en compte les seuls textes des 72 élèves tuteurs, émetteurs de critiques. Notre corpus est donc constitué de 576 textes appartenant au genre récit d'aventures et au genre texte descriptif et de 288 critiques portant sur chacun des genres considérés ${ }^{6}$.

\subsection{Textes informatifs-explicatifs et tâches proposées}

La première séquence de travail consacrée aux textes scientifiques explicatifs a débuté par une phase d'initiation à l'écriture de textes appartenant au genre et à l'élaboration de critiques destinées à aider des correspondants à améliorer leurs textes. Pour le reste, toutes les séquences se sont déroulées sur un modèle identique, débutant par une séance de sciences visant à construire l'explication scientifique d'un phénomène naturel selon une démarche expérimentale, préalablement à la mise en œuvre du dispositif de collaboration asymétrique. Les questions étudiées, choisies afin d'être à la portée des élèves mais suffisamment originales pour être ignorées de l'ensemble de ceux-ci, ont successivement été: " Comment un bulleur peut être utile à des poissons vivant dans un aquarium », « Pourquoi certaines îles et certaines régions du monde risquent d'être recouvertes par la mer dans quelques années », "Comment le cœur humain arrive à faire son travail qui est si compliqué (avec en particulier le rôle des valvules cardiaques) », « Pourquoi, en hiver, en France, certains animaux sont visibles et pourquoi d'autres disparaissent ». La deuxième séance était consacrée à écrire une première version du texte, la troisième à l'échange avec les correspondants (rédaction de critiques et de conseils), la quatrième à la révision du texte produit.

\subsection{Textes de fiction et tâches proposées}

Les séquences consacrées aux récits de fiction ont été organisées de la même manière, en articulation didactique avec le dispositif de collaboration. À quatre reprises, les élèves ont écrit des épisodes susceptibles d'être insérés dans un roman d'aventures pour la jeunesse ${ }^{7}$ dont ils venaient de lire des extraits. La première séquence a comporté une phase d'initiation à la récriture de récits d'aventures à partir de ressources empruntées à des fragments de romans appartenant au même genre, ainsi que d'initiation à l'élaboration de critiques et de conseils destinés à l'amélioration des textes des pairs. Pour le reste, les quatre séquences se sont déroulées en quatre séances, sur le modèle suivant: lecture de passages du roman et discussion collective sur ce qui tient le lecteur en haleine dans le passage ; lecture par l'enseignant de textes inducteurs et écriture individuelle de l'épisode supplémentaire destiné à être publié sur un site Web commun ; élaboration de critiques et conseils ; révision du texte. Quatre consignes ont été proposées : «Le héros, perdu dans la forêt sibérienne, va-t-il résister au froid et à la faim? Il s'organise pour vivre », "Le héros affronte une bête sauvage », "Le héros affronte une tempête », « Le héros cherche à échapper à des poursuivants ». Ajoutons que, dans toutes les classes, un travail de lecture a été conduit en parallèle sur d'autres romans d'aventures représentatifs du genre.

\section{Les élèves et les genres : analyse du corpus}

Le corpus a été analysé à partir des caractéristiques des genres présentées plus haut. Nous avons retenu des critères (i) communs aux deux genres - longueur du texte mesurée par le nombre de propositions sémantiques, cohérence de l'enchaînement des informations (isolées vs. liées), adéquation thématique et référentielle au sujet traité - (ii) spécifiques au genre explicatif - valeur épistémique (vrai / faux), causalité du monde physique, atemporalité et mise à distance généralisante - et (iii) spécifiques au genre roman d'aventures - vraisemblance, non contradiction, causalité intentionnelle, effets mimétiques et favorisant une implication affective du lecteur. 
L'analyse croisée des différents corpus nous permet de mettre au jour la manière dont évoluent la perception et la prise en compte, par les élèves, des caractéristiques génériques du texte explicatif et du texte de fiction. Nous nous intéressons donc aux processus de construction des connaissances implicites sur les genres par les élèves. Dans cette communication, nous retiendrons pour critères d'analyse ceux qui correspondent aux connaissances qui émergent dans les critiques émises et se stabilisent dans les textes révisés et les premières versions des textes ultérieurs. Nous classerons ces caractéristiques génériques en deux pôles : sémantique et linguistique-rhétorique ${ }^{8}$.

Notre méthode s'appuie sur les fondements théoriques de la psycholinguistique cognitive (McNamara, Floyd, Best \& Louwerse, 2004); elle recourt à une analyse sémantique reposant principalement sur les concepts d'information et de proposition sémantique, d'après les principes de l'analyse prédicative (Kintsch, 1974). En ce qui concerne le second pôle, nous avons eu plus particulièrement recours à des concepts issus de la linguistique de l'énonciation (Kerbrat-Orecchioni, 2002) et de la pragmatique, notamment le concept d'effet (Barthes, 1968).

Les textes que nous présentons dans le cadre de notre analyse qualitative, en section 3 de cette étude, ont été sélectionnés dans la mesure où ils constituent des illustrations corroborant les données quantitatives analysées dans une étude récente (Crinon \& Marin, 2010, soumis).

\subsection{Le pôle sémantique}

\subsubsection{Choisir les informations pertinentes ${ }^{9}$ (univers fermé vs. univers ouvert)}

Dans la plupart des textes des deux genres, des informations manquent dans la première version, surtout en début d'année.

Concernant le genre explicatif, les manques correspondent à une explication incomplète ou à un raisonnement lacunaire. C'est le cas du texte initial de Marin écrit lors de la première séquence, portant sur le fonctionnement du bulleur dans un aquarium :

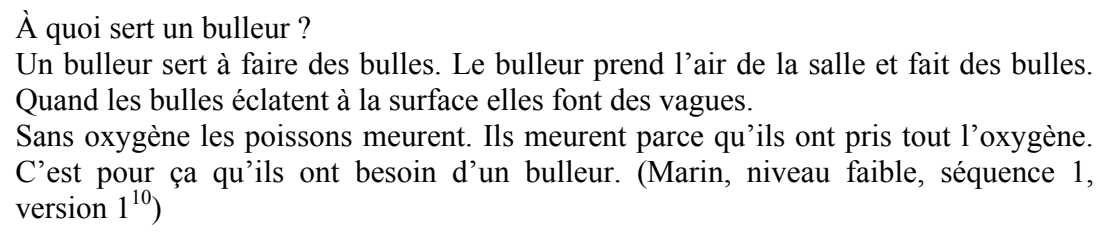

Or, cet élève, lorsqu'il se trouve en situation de critique, conduit, par ses questions ciblées, son interlocutrice à compléter les informations figurant dans son texte et le raisonnement afférent :

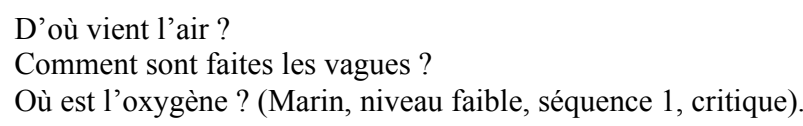

En réinvestissant les conseils à autrui et les informations lues et nécessaires à l'élaboration d'un texte à visée scientifique, il construit progressivement les enjeux du genre explicatif, mais n'améliore sur le moment que partiellement son propre texte en situation de récriture ${ }^{11}$ :

À quoi sert un bulleur?

Un bulleur sert à faire des bulles d'oxygène pour que les poissons respirent. [ $>\mathrm{Le}$ bulleur prend l'air de la salle et fait des bulles. Quand les bulles sortent, elles sont grandes et quand les bulles remontent, elles rapetissent.] Quand les bulles éclatent à la surface elles font des vagues.

Sans oxygène les poissons meurent. Ils meurent parce qu'ils ont pris tout l'oxygène. C'est pour ça qu'ils ont besoin d'un bulleur.

[Le bulleur prend l'air de la salle et fait des bulles. Quand les bulles sortent, elles sont grandes et quand les bulles remontent, elles rapetissent.] 


\section{Le bulleur est composé de : fragmenteur, tuyau en plastique et d'une pompe à} air. (Marin, niveau faible, séquence 1 , version 2)

Le deuxième texte de Marin en séquence 1 témoigne de sa difficulté à choisir les informations qui conviennent. Certes, sa critique du texte de Clotilde montre qu'il perçoit les informations manquantes. Mais il cherche à rendre son propre texte exhaustif en restituant des informations de la séance de science qui s'avèrent inutiles - la description de l'appareil - car non centrées sur l'explication demandée. En même temps l'explication elle-même reste lacunaire, implicite : il n'est pas précisé que l'oxygène est mélangé à l'eau grâce aux bulles et aux vagues produites à la surface de l'eau.

Le même élève, dans la séquence 3, mobilise des explications plus complètes et décrit méthodiquement le phénomène, dès sa première version :

Le corps humain et le cœur

Dans le corps il y a le cœur. Le cœur sert à vivre. Il y a des tuyaux qui relient le cœur à des organes.

Le sang passe par les tuyaux et va dans le cœur. Si le cœur ou les organes ne sont pas alimentés par le sang, on peut mourir.

Le cœur est entouré d'un muscle qui le contracte et décontracte. À l'intérieur il y a du sang. Quand ça se contracte, les valvules s'ouvrent et le sang part.

Les vaisseaux sanguins sont les tuyaux. Si le sang va à contresens, il peut percer les vaisseaux sanguins. Mais grâce aux valvules, le sang ne peut pas.

Conclusion: les valvules sont comme des portes qui s'ouvrent et se referment. Le cœur contrôle aussi les muscles comme le biceps, la cheville, etc.

Quand on court, notre cœur bat plus vite, donc le sang se déplace plus vite. Quand on ne respire plus notre cœur ne bouge plus, donc on peut mourir. (Marin, niveau faible, séquence 3 , version 1 )

Ce texte, comme le précédent, est encore marqué par une difficulté à procéder au choix des informations pertinentes, c'est-à-dire à la fois vraies, répondant à la question et reliées causalement aux autres informations du texte pour construire la chaine explicative. Il comprend des informations approximatives - le sang perce les vaisseaux - et hors sujet - les trois dernières phrases, qui relèvent d'un désir d'être exhaustif. En revanche, cet élève propose une chaine causale complète rendant compte de la complexité du rôle des valvules.

Ainsi, lors de l'écriture d'un texte explicatif, un premier obstacle est constitué par le choix des informations pertinentes, au sein de l'univers fermé des connaissances fournies - et mémorisées - sur le sujet : il s'agit d'une part de renoncer à présenter l'ensemble des informations disponibles (ne pas en dire plus que nécessaire) et d'autre part de donner l'ensemble de ce qui va permettre au lecteur de construire la cohérence causale de sa représentation du phénomène à expliquer.

Dans le cadre du genre récit d'aventures, les choix d'informations opérés par ces scripteurs novices nuisent très rarement à la cohérence causale de la situation, mais rapprochent les textes produits de sommaires, plutôt que de récits, notamment en ce qu'ils ne permettent pas de créer l'illusion mimétique. Les critiques des romans d'aventures ne proposent pas d'ajouter des éléments qui manqueraient à l'enchaînement des états - notamment des situations initiales et finales -, des actions, des événements, puisqu'on constate rarement ce type de manque. Ils proposent de créer des effets : effets mimétiques (descriptions d'objets, de paysages, d'états mentaux, de séquences d'actions), effets liés au suspense.

Ainsi Dany demande :

Comment allume-t-il le feu ? Comment se fabrique-t-il sa tente ?

Comment mange-t-il le tétras ? De quelle rivière s'agit-il ? Comment tue-t-il l'ours ?

(Dany, niveau fort, séquence 1 , critique)

Dès la version 2 de la séquence 1, qui suit l'émission de ces conseils, Dany multiplie les informations contribuant à l'effet d'univers :

Vassioutka va chercher des bouts de bois pour allumer le feu avec (ses) des silex et garde des allumettes. Quand il allume le feu, il se fabrique un arc avec des bouts de 


\begin{abstract}
bois et une corde pour tuer les oiseaux qui passent. (Et) Il a coupé quelques feuilles pour faire le matelas. Il prend (des jours pour fabriquer une petite tente) aussi plusieurs branches et des feuilles de sapin pour faire le toit. Il utilise ses feux pour faire des signaux de fumée pour faire fuir les bêtes. Il coupe le pain en plusieurs morceaux. Pour faire un couteau, il prend des bouts de bois et casse la pointe pour faire une forme de couteau. S'il rencontre un sanglier; il cache son odeur et lance le couteau sur la tête du sanglier et il meurt. Des fois, il va chercher des fruits. Il va aussi chercher des graines. Il a appris à rôtir ses graines en les plaçant sur des pierres tout contre le foyer. Il fabrique également des bols à partir du bois. Un jour, il a croisé un chasseur qui a bu. Vassioutka s'est caché et a tué le chasseur avec son arc. Il est triste des fois de ne pas voir sa mère.
\end{abstract}

Lors de la première version d'écriture de la séquence 2, Dany propose un récit d'emblée plus riche en informations, de nature à faire vivre la scène :
Vassioutka a très peur. Il chargea son fusil, il reste près du feu. Il entendit les buissons bouger à toute vitesse. Il transpire beaucoup et dit : " Montrez-vous, sinon je tire », en étant très inquiet; puis il entendit râler, c'était un lynx mais lui ne savait pas que c'était un lynx. Il prend un gros bout de bois, le met sur le feu. Avec ça il faut une torche. Il voit des grosses oreilles, de la bave qui coule. Il voit de longues griffes, puis il voit des yeux verts sauter sur lui. Il tira deux coups " pan! », touché, mais le lynx était encore en vie. Il le griffa sur la joue. Il prit la torche, il le brûla sur sa face, reprit son fusil et deux balles dans le cœur. (Dany, niveau fort, séquence 2, version 1)

La mise en scène d'un animal sauvage, familière à l'univers fictionnel des élèves de cycle 3 laisse place, lors de la séquence suivante, à une consigne d'écriture d'une plus grande difficulté, invitant à décrire le héros en proie aux éléments déchainés. Pourtant la progression dans la qualité du texte de Dany se poursuit par l'insertion d'expansions qui rendent la scène dynamique :

\begin{abstract}
Vassioutka courut à toute vitesse en espérant trouver un abri. Il avait trouvé une grotte. Il courut aussi vite vers la grotte mais le tonnerre fit des éclairs sur les arbres. Il tomba à terre, fit trembler le sol et bloqua l'entrée de la grotte. Vassioutka avait très peur et il y avait beaucoup de pluie, de tonnerre, d'orage. Et les nuages tout noirs lèvent, emportent les feuilles, râpent le long de l'eau, montent dans les branches des nids d'oiseaux. Vassioutka avait tellement peur qu'il pleura. Il y avait un petit passage entre les arbres écroulés et la grotte. Il se dit: «Bon, je suis courageux, j'irai ! » Il prit son sac, le mit au-dessus de sa tête et courut vers le trou et passa. Il était dans la grotte. (Dany, niveau fort, séquence 3, version 1)
\end{abstract}

De même Audrey indique-t-elle à l'adresse d'Anthony :

Il n’y a pas de poursuite dans ton texte. [...] Pour la poursuite tu pourrais mettre : « Il s'engouffra dans la forêt de l'autre côté, se cogna aux basses branches des épicéas ». (Audrey, niveau fort, séquence 4, critique)

Audrey fait partie des élèves dont le récit, en fin d'année, donne à voir et à entendre les scènes qu'elle compose, appliquant elle-même les conseils formulés à mesure qu'elle se construisait un modèle du genre roman d'aventures. En même temps qu'elle prend conscience qu'écrire un roman, c'est multiplier les notations qui permettent d'évoquer un univers, elle recourt à des métaphores et comprend le rôle unificateur du point de vue narratif; le lecteur découvre le spectacle par les yeux du personnage, et ce spectacle possède une fonction dans le récit, par la menace qu'il fait peser sur le personnage :

\footnotetext{
Vassioutka était terrifié par ce spectacle terrifiant. Les nuages étaient d'encre, la pluie croulait comme si des bouts de plomb tombaient du ciel. Des arbres gigantesques se courbaient sous les assauts de furieuses bourrasques. (Audrey, niveau fort, séquence 3 , version 1)
}

Ainsi, le monde ouvert du roman d'aventures suppose des amplifications, des expansions narratives et des descriptions... et donc des ressources nombreuses où aller puiser. 
Par opposition à cette ouverture des possibles dans un récit de fiction, le domaine des connaissances où puiser pour expliquer un phénomène scientifique est, nous l'avons dit, clos. La concision fait partie des critères du genre explicatif. Y contribue la recherche du mot juste et univoque, non remplaçable par un synonyme. Ainsi Erwan invite-t-il, par sa critique, à adopter un lexique précis :

Tu peux rajouter le mot oxygène après le mot bulle. Sinon, ton texte est bien. (Erwan, niveau fort, séquence 1 , critique)

Cette prescription conduit Erwan à substituer lui-même à «air » le terme plus précis «oxygène » dès l'introduction de son texte :
Le bulleur
Pourquoi le bulleur est nécessaire pour les poissons vivant dans un petit aquarium ?
(Les poissons) de petits animaux aquatiques ont besoin d'un bulleur pour survivre. Le bulleur est un mécanisme qui permet à l'(air) oxygène de se dissoudre dans l'aquarium. Le bulleur a un moteur, un tuyau en plastique et un fragmenteur, petit appareil qui transforme l'oxygène en bulles. C'est par là que passe l'oxygène, gaz invisible qui flotte dans l'air.
Les poissons qui sont en liberté (ont) obtiennent de l'oxygène grâce aux vagues qui absorbent de l'(air) oxygène qu'il y a dans l'air mais ils ont aussi les branchies qui les aident à respirer comme nous avec nos poumons. (Erwan, niveau fort, séquence 1, version 2)

Les conseils d'Erwan portent de manière récurrente sur la nécessité d'utiliser le terme précis; ainsi il propose à Mame :

Remplace le mot "barrières" par "des valvules". (Erwan, niveau fort, séquence 3, critique)

et à Hugo, notamment :

Remplace le mot "tunnel" par le mot "tuyau".

Remplace le mot "porte" par le mot "valvule". (Erwan, niveau fort, séquence 3, critique)

En cela la quête du terme juste fait partie des apprentissages suscités par le dispositif à destination des élèves tuteurs dont les textes évoluent en relation avec la qualité des critiques émises. Cette exigence à l'égard de la précision du vocabulaire d'autrui devient une autocontrainte grâce à laquelle Erwan perçoit, construit et mobilise les critères du genre texte explicatif, ainsi que le montre la première version du texte écrit en séquence 4 :

En France, en hiver, certains animaux sont visibles d'autres non, comme les ectothermes - les reptiles et les batraciens. Ils ne sont pas visibles car leur corps varie selon la température extérieure, donc, ils sont en léthargie : leur organisme est ralenti. Or, cela leur est bien utile car c'est comme s'ils avaient fait des réserves. C'est pour cela qu'on ne les voit pas... Ils sont cachés sous les rochers.

Cela ne veut pas dire qu'on ne voit pas d'animaux. Il existe d'autres espèces d'animaux, les endothermes comme les mammifères et les oiseaux qui sortent pour chercher de la nourriture, car contrairement aux ectothermes, leur corps reste à température constante, donc, leur organisme ne ralentit pas, ils ne sont pas en léthargie. Cela veut dire qu'ils sont visibles en hiver et ils ont beaucoup besoin de nourriture pour garder leur chaleur.

C'est pour cela que certains animaux sont visibles en hiver et d'autres non. (Erwan, niveau fort, séquence 4 , version 1 )

La précision du lexique scientifique employé rend particulièrement efficaces les jeux d'emboîtement des termes génériques et spécifiques qui confèrent à cet élève une attitude de surplomb par rapport aux notions mobilisées. 


\subsubsection{Vérité et vraisemblance}

Poursuivant l'observation des étapes de la construction du genre chez ces élèves de cycle 3 à l'aune des critiques qu'ils formulent en tant que tuteurs, nous avons analysé leurs écrits du point de vue des catégories du vrai et du vraisemblable.

Dans le texte explicatif, la vérité scientifique est perçue dans son univocité et les critiques pertinentes le donnent à lire sans ambiguité. L'activité de critique est étayée par la lecture des textes de plusieurs correspondants, ce qui permet aux élèves de niveau faible d'apprécier la valeur de vérité des informations contenues dans ces textes afin de pouvoir rédiger leurs critiques en dépit même des erreurs initiales dans la compréhension ou la mémorisation du contenu de la leçon de sciences. Ainsi, Mélanie adresse-t-elle successivement ces critiques à Anaïs :

Tu as parlé de tout. Je trouve ça très bien.

Mais les bulles d'air ne vont pas exploser.

Le bulleur n'est pas un simple objet. Il n'y a pas de « mini-vagues ».

Il faudrait expliquer mieux. (Mélanie, niveau faible, séquence 1, critique)

C'est très bien mais tu as oublié de dire que le cœur bat 70 fois par minute.

$\mathrm{Tu}$ as raison, le cœur est un muscle mais tu as oublié de dire qu'il était creux.

Le sang contient de la nourriture et de l'oxygène. (Mélanie, niveau faible, séquence 3, critique)

En début d'année, son premier texte présente une série d'informations qui ne répondent pas aux critères de la vérité scientifique, qu'il s'agisse des informations elles-mêmes ou de leur mise en relation.

Les poissons ont besoin d'oxygène grâce au bulleur.

Ça ne sert pas juste à faire beau, ça sert aussi à récupérer l'air qui est dans la salle qui l'amène dans le tuyau. Comme ça le poisson ou les poissons peuvent respirer comme nous. Simplement grâce à l'oxygène mais je pense que même si l'aquarium est petit, ils respirent toujours comme s'ils étaient dans la mer. Simplement que dans l'aquarium c'est plus petit et ils arrivent mieux à trouver à manger ou à respirer. J'ai remarqué que le tuyau fait plein de petites bulles qui vont jusqu'en haut de l'aquarium qui rend ça intéressant. (Mélanie, niveau faible, séquence 1, version 1)

Les progrès dans son activité de critique vont de pair avec la compétence à identifier et mettre en relation les informations vraies, de sorte qu'à la fin de l'année, cette élève est capable de produire un texte acceptable sur ce plan :

Il y a des animaux, comme l'hirondelle qui passent l'hiver en Afrique, donc, ils disparaissent en France, tout l'hiver. Il y en a qui restent et continuent à chercher leur proie, comme le renard, le lapin...

Il y en a qui restent tout l'hiver, comme les moineaux...

Plusieurs animaux disparaissent car ils ne supportent pas l'hiver donc il y en a qui se cachent, d'autres, vont dans un autre pays pour y passer l'hiver et reviennent l'été.

Il y a ceux qui restent l'hiver et cherchent leur proie, ceux qui se cachent tout l'hiver dans un autre pays et reviennent l'été. Puis, ceux qui meurent. Ils sont visibles parce qu'ils sortent manger et pour se réchauffer ils mangent beaucoup. (Mélanie, niveau faible, séquence 4 , version 1 )

En revanche, le genre récit d'aventures, par son inscription dans la fiction, suppose un glissement du vrai au vraisemblable, ce qui ne va pas sans difficulté pour certains. Aurélie commente ainsi :

Je trouve ton texte un peu trop tarzan, car tu dis qu'il prend une liane, est parti dans un autre arbre. (Aurélie, niveau faible, séquence 2, critique)

La difficulté de sa partenaire à circonscrire le vraisemblable est mise au jour par l'émettrice de critiques qui signale une référence intertextuelle qu'elle juge inopportune. Certains élèves condamnent ainsi la dérive des codes réalistes des romans d'aventures " géographiques » qui leur ont été proposés dans la classe vers l'imaginaire des contes ou de la fantasy: 
Ce à quoi Issa, l'élève à qui sont adressées ces critiques, répond : "C'est un roman », montrant ainsi les limites de son appréhension du genre récit d'aventures. L'invraisemblance est aussi pointée en relation avec les connaissances sur le monde relevant de la culture personnelle de l'élève. Écrire un roman d'aventures oblige à utiliser des informations véridiques sur la région où se situe l'action, ce que ne manque pas de relever Hugo, jugeant le texte d'un de ses pairs :

Je trouve bizarre qu'il y ait des bananes en forêt sibérienne. (Hugo, niveau faible, séquence 2 , critique)

À la fin de l'année, si ces invraisemblances se retrouvent encore en de rares occurrences dans les textes des élèves récepteurs de conseils, elles ont totalement disparu des écrits des élèves tuteurs. Ces derniers mentionnent des animaux ou décrivent une végétation en cohérence avec le cadre spatial de leur récit.

\title{
3.1.3 Particulariser ou généraliser
}

Le texte narratif suscite des situations particulières et originales et donne à voir au lecteur un monde par le jeu des «effets de réel». C'est ce que les critiques soulignent, accompagnant, chez ceux qui les formulent, un recours à de tels effets dans leurs propres textes.

Vuong conseille ainsi à Bilel :

Il faut plus de poursuite. Et tu peux écrire ce que ressent Jeremy : «L'enfant se mit à courir, moitié hurlant, moitié pleurant ». (Vuong, niveau fort, séquence 4, critique)

La particularité de la critique consiste à s'adapter au texte commenté pour s'inscrire dans sa dynamique. En effet, si l'élève tuteur prend à son compte les conseils qu'il donne, et réalise une meilleure performance d'écriture grâce aux ajouts de la version 2, il s'y contraint en spécifiant les images insérées au contexte de la première version d'écriture.

\begin{abstract}
Jeremy courait toujours mais il commençait à être fatigué. Soudain, il rencontra une veuve noire. Il resta pétrifié, incapable du moindre mouvement, comme si ses jambes venaient d'être tranchées d'un magistral coup de faux. Il paniqua et sauta. L'araignée se mit à courir. Jeremy se retourna. Lorsqu'il ne vit plus l'araignée, il continua à marcher. Tout à coup, il marcha sur quelque chose de bizarre. Alors il se dit que l'araignée qui avait disparu était sur sa route. Il avait très peur. Il regarda sur le sol l'araignée. Il se mit à courir. Quelques minutes après il était essoufflé, il reprit sa course. Soudain, il glissa, il se prit un rocher. Heureusement qu'il glissa car juste devant, il y avait un marécage boueux bourré de crocodiles. Il devait le traverser sinon les gardes le rattraperaient. Alors il remarqua quelque chose, le dos des crocodiles était tout plat. Il se mit à sauter sur leur dos. Tout à coup, il trébucha sur un des crocodiles, il y avait une liane juste au-dessus. Il s'agrippa sur la liane et de justesse il était sur l'autre rive. Jeremy entendit soudain les gardiens. Prêt à détaler comme un lapin, il courut, les gardiens étaient juste derrière lui, alors il prit un caillou, le jeta sur les gardes. Soudain, il y eut une grotte. Il se réfugia. Les gardes rentrèrent et soudain un tigre de Tasmanie poursuivit les gardes. Jeremy était dans un creux. Le tigre poursuivit les gardes. Jeremy reprit sa route. Déterminé de ne pas retourner dans la plantation, l'évadé cria de joie car heureux d'être échappé des gardiens. (Vuong, niveau fort, séquence 4, version 2)
\end{abstract}

La prégnance de l'effet de réel tient ici à la création d'un univers singulier et visuel où est donnée à voir la particularisation de la situation décrite.

En revanche, le genre texte explicatif demande de généraliser des explications à partir d'une expérience contextualisée et à les abstraire. Au cours de la première séquence, les élèves ont observé successivement le fonctionnement d'un bulleur dans un véritable aquarium et, sur un support papier, un schéma représentant la diminution de la taille des bulles et le mouvement de l'eau. Or, certains élèves qui n'ont 
pas compris ces enjeux du genre explicatif font explicitement référence, lors de leur première version d'écriture, au contexte de la situation d'apprentissage :

Dans l'aquarium il y a des bulles parce que les poissons respirent dans l'eau et ils ne peuvent pas vivre sans oxygène. Et dans les bulles il y a de l'oxygène. Les bulles sont de plus en plus petites. L'oxygène est dans l'air et après il part dans l'eau.

Dans ce schéma en haut il y a de l'air et les petits points c'est de l'air. Dans le fragmenteur part de l'air et il le transforme en bulles (Pirasannah, niveau faible, séquence 1 , version 1 )

La présence même du mot « schéma » montre que Pirasannah peine à décontextualiser son raisonnement de la situation dans laquelle ont été produites les connaissances scientifiques, de même qu'au début de son texte, la mention de l'aquarium et des bulles renvoie à l'observation effectuée et non à un discours généralisant mettant en scène des savoirs. Les obstacles auxquels est confrontée cette élève sont concomitants de sa difficulté à prodiguer des conseils constructifs lors des séquences 1 et 2 :

Il est bien ton texte. Je n'ai rien à te dire. Il n'y a que ça à dire et je n'ai pas de remarques. (Pirasannah, niveau faible, séquences 1 et 2, critiques)

En revanche, à partir de la troisième séquence, cette élève perçoit plus clairement la nécessité de se déprendre de la narration d'une observation ponctuelle pour inscrire son texte dans le genre scolaire attendu. Lors de la troisième séquence, les élèves avaient procédé à une expérience afin de comprendre, par analogie, les contraintes du système de circulation du sang : si l'on exerce une pression sur un tube de dentifrice qui a plusieurs ouvertures, la pâte sort par toutes les ouvertures. Écrire le texte demandé supposait une décontexualisation de la situation expérimentale observée pour énoncer les principes généraux régissant le fonctionnement du cœur. Cette fois, Pirasannah réussit à se détacher du contexte de la situation d'apprentissage et ne fait pas référence à l'expérience réalisée avec le tube de dentifrice :

\begin{abstract}
Le cœur arrive à faire son travail qui est si compliqué, parce que le sang vient dans le cœur et les organes et il repart dans les organes. Les portes que le sang ouvre dans le cœur. Le sang des organes part au cœur, et le sang du cœur repart dans l'organe. Et quand il est rentré, il ferme la porte et il fait «boum boum », parce qu'il y a deux portes, une porte qui rentre et une deuxième porte qui se ferme. Le sang part et revient. La porte s'appelle «valvule». Le muscle serre le cœur. Quand on court, le sang se précipite dans les organes et les valvules se ferment et ouvrent et les valvules font « boum boum ». (Pirasannah, niveau faible, séquence 3, version 2)
\end{abstract}

Il est intéressant de noter qu'en revanche, lors de son activité de critique, dans le cadre des conseils émis à destination de Manon, Pirasannah fait allusion à l'expérience menée en classe pour faire comprendre par analogie le fonctionnement cardiaque, partiellement présenté par sa camarade :

Le cœur fait un travail difficile. On peut percer le tube de dentifrice comme le cœur. Dans le tube quand on fait un autre trou il peut sortir par les deux côtés. (Pirasannah, niveau faible, séquence 3 , critique)

On peut noter les progrès conduits parallèlement dans l'élaboration de critiques et dans le processus de décontextualisation dans le genre explicatif ${ }^{12}$. L'appropriation de ce critère du genre bénéficie du travail d'élaboration et de prise de distance que suppose l'activité de critique, qui favorise le choix d'informations généralisantes et abstraites de préférence à des informations référées à la situation vécue.

\title{
3.2 Le pôle linguistique et rhétorique
}

\subsubsection{Exprimer et créer l'émotion vs. bannir l'émotion}

La place de l'émotion et des procédés rhétoriques qui la suscitent constitue un autre axe qui différencie ces genres. Valorisée dans le texte narratif, l'émotion est bannie du texte explicatif scientifique. La progression dans l'appréhension de ces codes recouvre celle de la qualité des textes produits. 
Nombre de commentaires des auteurs de critiques portant sur les textes de fiction concernent la psychologie des personnages et les effets qui en découlent pour le lecteur. Leur mention dans les critiques croît au fil des séquences. Ils concernent tout particulièrement la manifestation de sentiments et des sensations des personnages, élément qui concourt à créer des émotions chez le lecteur. La récurrence des conseils visant à faire introduire le suspense dans la version ultérieure des textes lus va de pair avec une appropriation des procédés. Prenons l'exemple de textes de Yannick qui tire un grand parti de son travail de critique de ce point de vue. Son premier texte joue sur l'humour, et non pas sur ce qu'une situation désespérée peut inspirer tout à la fois au personnage et au lecteur :
Vassioutka cherche du gibier pour le faire griller mais la nuit, il se dit : « Je le ferai demain. » Le lendemain matin, il prit son fusil et partit chasser.
- Cui cui, gros tétras où es-tu? dit Vassioutka. Puis il entendit un bruit bizarre. «Ah! qu'est-ce que c'est? », hurla-t-il. C'était son ventre. Il se trouva sur un nid au milieu de la forêt. "Le voilà !». Il tira en visant la tête. Et il le mit dans son sac. (Yannick, niveau fort, séquence 1, version 1)

En séquence 4, il critique le texte de Jérémie en ces termes qui invitent à accroître les émotions liées à la situation :

Dans ton texte Jeremy n'a pas peur. Tu peux dire : « Jeremy effrayé de peur assomma le crocodile $"$.

Tu ne parles pas beaucoup des gardiens. Tu peux dire : «Les gardiens avançaient, avançaient si vite qu'ils crurent voir une ombre ». (Yannick, niveau fort, séquence 4, critique)

Aussitôt après, Yannick devient le premier bénéficiaire de ses propres conseils, qu'il réinvestit plus habilement qu'il ne les a exprimés, dans la deuxième version de son texte :

\begin{abstract}
Jérémy (avança, avança) traversa un marigot mais il se faisait stopper par une très grosse toile d'araignée. Puis effrayé, il prit un bâton pour casser la toile mais au moment où il se plia il sentit une démangeaison comme si quelque chose montait sur son dos. (Il se dit c'est mon hallucination) Mais pendant ce temps Cuir-de-Vache et le surveillant entendirent un sifflement mais continuent à avancer. Puis Thompson voulut aller plus vite (imiter) imitant Tarzan. Il sauta puis attrapa une liane puis une deuxième. À la troisième il tomba. Ce n'était pas une liane mais un serpent, un serpent affamé même très affamé. Cuir-de-Vache dit à Thompson :

«Ne bouge surtout pas.

— Ou... ou... oui... ch.. ch... chef » dit Thompson effrayé.

Effrayé, il courut si vite (qu'il voyait une silhouette. Ce n'était pas une silhouette mais Jérémy) que les gardiens ne voyaient plus, Jérémy. (Yannick, niveau fort, séquence 4 , version 2 )
\end{abstract}

L'introduction du jeu des émotions semble ainsi se constituer en l'un des éléments fondateurs du genre récit d'aventures. De la première à la dernière séance, les textes écrits par Yannick montrent la progression dans la mention des sentiments en lien avec l'appropriation de ce nouveau critère de genre.

En revanche, les indices d'une quelconque émotion sont proscrits dans les textes explicatifs, ce que peinent à comprendre beaucoup d'élèves, qui prennent l'information fournie par la séance de sciences dans un rapport premier et affectif au monde. C'est le cas de Kenza; lors de la séquence 2 portant sur la montée des eaux, dans l'introduction et la conclusion, elle déplace son discours de l'objectivité scientifique à la subjectivité compassionnelle qui le disqualifie :

Figurez-vous que les habitants des atolls* courent de grands risques! Car petit à petit, la mer monte, monte, monte, et risque d'envahir leurs îles ! Et si malheureusement ce drame arrive, les pauvres habitants devront quitter leurs îles. Parce qu'en été, avec la chaleur, l'eau se dilate* petit à petit, millimètre par millimètre, et détruit les atolls.

* atolls : petites îles plates sans montagnes ni collines au milieu de l'océan.

* dilate : prendre plus de place. (Kenza, niveau fort, séquence 2, version 2) 
L'irruption de l'émotion en version 2 intervient alors qu'aucun des textes que Kenza a été amenée à lire ne contenait ce travers. Pourtant, les critiques émises par Kenza n'incitent jamais ses pairs à recourir à des modalisations affectives lors de la révision de leur texte explicatif et, à la fin de l'année, son style devient plus neutre à cet égard :

\author{
L'hiver et les animaux \\ Figurez-vous qu'il y a deux types d'animaux. \\ Il y a les animaux ectothermes* et les animaux endothermes* d'après les biologistes*. \\ *ectotherme : animaux qui meurent en hiver \\ exemple : les coccinelles \\ *ectotherme : animaux qui se cachent pendant l'hiver. \\ exemples : les lézards \\ *endotherme : animaux qui émigrent en hiver. \\ animaux qui restent pendant l'hiver. exemple : chiens \\ *biologistes : spécialistes qui observent les êtres vivants. \\ Donc il y a moins d'animaux en hiver, en France car il y a des animaux ectothermes, \\ ce sont ceux que l'on ne voit plus, mais on voit quand même des animaux car il y a \\ les endothermes, ceux qu'on voit. (Kenza, niveau fort, séquence 4, version 1)
}

Alors même que la mort des animaux ectothermes aurait pu être de nature à faire surgir des éléments de pathos, il n'en est rien dans ce texte, pas plus que dans ceux des autres élèves émetteurs de critiques. Le dispositif de travail d'élaboration de critiques semble ainsi permettre à long terme d'éliminer des textes explicatifs scientifiques l'expression de l'émotion.

\title{
3.2.2 Chercher l'effet énonciatif vs. chasser l'effet énonciatif
}

Enfin, on observe une évolution dans l'usage des effets énonciatifs, proscrits dans le texte explicatif et au contraire prescrits dans le texte narratif.

Il a déjà été souligné dans d'autres études que la prise à témoin du lecteur, l'intrusion de l'auteur et de sa subjectivité, valorisées dans le récit (et tout particulièrement dans la rédaction scolaire) sont volontiers utilisées par les élèves dans leurs textes scientifiques (Bautier, Manesse, Peterfalvi \& Vérin, 2000). Cette importation d'effets énonciatifs apparaît bien dans le texte d'Élodie sur le fonctionnement cardiaque (séquence 3$)^{13}$.

Le cœur et son travail

Le cœur a un travail très compliqué. Vous voulez que je vous explique ? Alors, voilà.

Le coeur a des tuyaux qu'on appelle des vaisseaux sanguins. Les vaisseaux sanguins servent à faire circuler le sang vers les organes et repartent vers le cœur. Le cœur est un sac qui se vide de sang et qui se remplit de sang. Le cœur bat 70 fois par minute et quand le cœur bat, ça fait « boum boum boum boum ». Comme si c'était des portes. Il peut arriver un grave accident. Lequel ?

Imaginez votre cœur. Si le sang se rencontre, il va arriver un accident et on peut mourir. Pour ne pas mourir, il y a des valvules, pour ne pas avoir d'accident. Mais il peut toujours en arriver des accidents. (Élodie, niveau faible, séquence 3, version 1)

L'effet énonciatif consiste ici à apostropher le lecteur par le biais de la fonction phatique et notamment de questions oratoires, à introduire des onomatopées qui mettent en scène l'expressivité du narrateur, ellemême rehaussée par la cataphore pronominale de la dernière phrase («il peut toujours en arriver des accidents »). De plus, la référence à l'univers quotidien fait basculer le texte vers le genre narratif.

La lecture des textes de ses pairs joue, chez Élodie, un rôle d'acculturation à la rhétorique du genre explicatif, de sorte que son texte se débarrasse de certaines scories sans pour autant toutes les éliminer :

Pourquoi certains animaux sont bien visibles et pourquoi d'autres disparaissent?

Je vais vous expliquer. Lisez bien.

Ectotherme

Un animal ectotherme? 
Didactique et enseignement, français langue maternelle, français langue seconde DOI $10.1051 / \mathrm{cmlf} / 2010207$

Un animal ectotherme est un animal qui meurt et qui disparaît et qui se cache et aussi ils émigrent et ils sont gelés et meurent de froid.

Endotherme

Un animal endotherme est un animal qui ne meurt pas et qui résiste au froid et qui ne disparaît pas et aussi c'est un animal qui reste. Voilà.

Biologiste

Un biologiste c'est quelqu'un qui explique bien la vie des animaux.

Alors voilà pourquoi les animaux sont bien visibles et d'autres disparaissent.

(Élodie, niveau faible, séquence 4, version 1)

Ce texte est marqué par une régression des commentaires personnels. Si une question rhétorique demeure (« Pourquoi certains animaux sont bien visibles et pourquoi d'autres disparaissent? ?), celle-ci joue un rôle didactique en reprenant les termes du problème scientifique à élucider.

À l'opposé, dans les textes narratifs, l'évolution va de textes où l'énonciateur s'efface vers des textes de plus en plus « évaluatifs », au sens où Labov (1978) emploie ce terme - l'auteur intervient pour souligner en quoi son histoire vaut la peine d'être racontée -, et de plus en plus « modalisés ».

L'exemple de Shirley nous permet d'appréhender les étapes de la construction de la représentation du genre récit d'aventures à cet égard. Son premier texte fournit une succession d'actions organisées chronologiquement sans aucun artifice énonciatif :

Vassioutka alluma un feu puis il ramassa des branches d'arbres et des feuilles épaisses. Il commença à construire une cabane, ensuite, il fit un lit en feuilles qu'il glissa à l'intérieur de la tente. Soudain, il s'arrêta parce qu'il avait faim ; il pensa à l'oiseau dans son sac. Vassioutka prit son oiseau avec quelques cônes de cèdre. Il dépluma l'oiseau, frotta les cônes les fit cuire. Vassioutka finit de manger, il éteignit le feu puis il s'endormit et il pensa que le lendemain il retrouverait son chemin. (Shirley, niveau fort, séquence 1 , version 1)

Le chemin parcouru entre la première et la dernière séquence permet d'appréhender la richesse et la diversité des compétences acquises dans la production de textes relevant du récit d'aventures :

Pendant ce temps, Jeremy continuait à escalader posément les pentes du premier contrefort de la Cordillère. À peine fini d'escalader le premier contrefort, le fugitif pouvait tomber dans des sables mouvants.

Cette forêt abritait énormément de sables mouvants, marigots, serpents et des veuves noires. Ce qu'il détestait prodigieusement.

Jeremy se rattrapa comme un équilibriste avant qu'il tombe dans les sables. Le garçon sauta par dessus. Quand il arriva derrière le sable mouvant, il vit le garage où on rangeait les motos, les voitures qui servaient à transporter les cannes à sucre. Jeremy eut l'intelligence exceptionnelle d'emprunter une moto pour fuir plus rapidement ses poursuivants. Mais avant Jeremy eut des fourmillements dans les jambes. Il enleva son pantalon et trouva une veuve noire qui faisait le marathon à la recherche des blessures, gorgée de sang. Le garçon l'attrapa et la jeta le plus loin. Après l'avoir jetée, il lécha sa piqûre.

Ses poursuivants étaient de plus en plus proche du « gibier ». Dogue s'arrêta et hurla de douleur. Thompson regarda et constata que Jeremy avait semé des graines toxiques. «Le gredin, il a semé des graines toxiques », cria Thompson. « Comment va-t-on faire? » interrompit le chef surveillant.

Jeremy entra, mit le casque de moto et surgit du garage. Il arriva juste devant ses poursuivants. C'est comme ceci que continua la course poursuite. Jeremy pleurait de joie. Il avançait comme un guépard sur sa moto. Il dérapa. Ses poursuivants s'arrêtèrent. (Shirley, niveau fort, séquence 4 , version 1)

Les anaphores nominales (« le fugitif»), (« du “gibier”») renforcée, pour la seconde, par l'emploi subtil des guillemets, crée un glissement du point de vue du narrateur à celui des poursuivants, et permet l'immersion du lecteur dans leur univers mental. Les modalisateurs ("pouvait », « énormément», «prodigieusement») indiquent le risque encouru par le héros et le fragilisent. Il est ainsi rendu éminemment vulnérable par la comparaison (« comme un équilibriste »). Le commentaire du jeune auteur 
narrateur sur son personnage (« eut l'intelligence ») le met aux prises avec les intentions malveillantes et murmurées de ses poursuivants : « comment va-t-on faire ? »). Les changements de point de vue narratif, les intrusions d'auteur, le suspense lié aux modalisations concourent à la qualité de ce texte, bien éloigné de la simple succession d'actions produite en début d'année scolaire.

Pourtant, ni cet élève, ni aucun autre ne formule de critiques évoquant la création de ce type d'effet. La complexité des réalisations textuelles est encore plus délicate à prescrire qu'à mettre en œuvre au stade développemental dans lequel se trouvent des élèves de la fin de l'école élémentaire. En revanche, la confrontation à des textes de pairs relevant du même genre et le constat de leurs défaillances au regard de textes de littérature de jeunesse consultés parallèlement semblent contribuer à construire chez les élèves tuteurs les critères liés aux effets énonciatifs, indépendamment du métalangage afférent. En reprenant à notre compte le postulat de Vergnaud (2003), nous pouvons noter que la forme opératoire de la compétence à créer des effets énonciatifs apparaît ici supérieure à sa forme prédicative.

\section{Conclusion}

Ainsi, à travers la prise en compte de ces critères associés en des formations discursives complexes, les critiques cernent progressivement les caractéristiques du genre visé, lequel est parfois explicitement nommé, par Shana, notamment :

« C'est bien car ça a vraiment le style d'un roman d'aventures. »

ou plus marginalement pour le genre explicatif, par Alexandre :

« Ton texte est explicatif. »

Et l'évolution des textes produits semble indiquer que caractériser les genres, non pas a priori, mais en interaction avec l'écriture, contribue à en intérioriser les codes et à être capable de les mobiliser en situation d'écriture. Ainsi, l'analyse comparative des textes produits dans les deux genres par les élèves nous conduit à affirmer que travailler l'écriture dans les genres à partir d'un dispositif de collaboration asymétrique ancré dans une situation didactique idoine permet aux élèves placés en situation réflexive de s'approprier et de mettre en œuvre progressivement les contraintes liées à des genres scolaires quasi antonymiques. S'y affrontent en effet des contraintes génériques régissant diversement la sélection des informations pertinentes et le choix des mots - monosémiques et précis $v s$. polysémiques et métaphoriques - la compétence à inscrire une pensée dans un processus de généralisation $v s$. de particularisation et le statut du vrai vs. du vraisemblable, de l'émotion et des effets énonciatifs.

Ces analyses mettent en évidence plusieurs obstacles rencontrés par les élèves de la fin de l'école primaire dans leur apprentissage des genres narratifs et explicatifs. Ils ne tiennent pas essentiellement à l'organisation des textes, mais à une série de caractéristiques spécifiques et à la confusion entre genres, qui semble les empêcher de relier ces caractéristiques aux intentions qui les fondent.

Mais les apprentissages que nous avons illustrés dans cette communication par des exemples emblématiques tirés de notre corpus peuvent-ils vraiment être attribués au dispositif didactique dans lequel ils ont eu lieu ? Plusieurs éléments qui n’ont pas encore évoqués ici nous incitent à le penser. La supériorité des progrès des élèves qui ont élaboré des critiques par rapport aux partenaires qui les ont utilisés laisse penser que le fait d'émettre des critiques a joué un rôle dans les progrès observés (pour une analyse statistique du corpus des textes explicatifs, voir Crinon \& Marin, 2008). En outre, la comparaison des données utilisées ici pour les récits avec celles recueillies dans une phase précédente de la recherche où n'avait pas été mené un travail systématique le lecture de romans d'aventures en même temps que le projet d'écriture, peut faire penser à l'importance des interactions lecture écriture dans l'apprentissage de l'écriture de fiction.

Nous formulerons donc l'hypothèse que ce qui a permis aux élèves de progresser dans leur pratique de ces genres, c'est la compréhension du rapport entre les procédés utilisés et l'activité cognitive sollicitée, l'intention cognitivo-discursive donc. Les élèves dont nous avons observé les productions acquièrent et mettent en œuvre des connaissances sur le genre qu'ils pratiquent, non pas dans le cadre d'un 
enseignement formel, mais dans une pratique d'écriture intégrée à l'enseignement d'une discipline. Celleci possède trois caractéristiques : l'élève écrit en fonction d'une intention, il a à sa disposition des ressources, il est mis dans une situation qui lui permet de prendre du recul et de théoriser les critères du texte visé. Comme le dit Olson (2008), écrire est une fonction, pas seulement un savoir faire. C'est bien sur la fonction de l'écrit que les élèves ont travaillé ici. Si les élèves ont de mieux en mieux distingué deux types d'énonciation (texte où l'auteur est présent et texte où l'auteur s'efface), ou encore deux rapports aux informations sources (saturer le texte pour créer un effet d'univers et s'en tenir aux informations strictement nécessaires à l'explication du phénomène), c'est parce que, dans un genre comme dans l'autre, le «je » du sujet scripteur et du sujet pensant, engagé dans une activité signifiante, était sollicité.

Remerciements : Les auteurs remerciement l'IUFM de l'académie de Créteil pour son soutien à cette recherche. Ils tiennent également à manifester leur gratitude aux enseignants des classes participantes, aux directeurs et aux équipes de circonscription et leur dette aux autres chercheurs de l'équipe qui ont apporté leur concours à la conception du programme de recherche, au travail dans les classes ou à la mise en forme des corpus : F. Amouri, P. Avel, I. Ayab, A. Cautela, G. Ferone, D. Legros, A. Maillard, P. Richard.

\section{Références bibliographiques}

Adam, J.-M. (1997). Les textes : types et prototypes. Paris : Nathan Université.

Bakhtine, M. (1984). Esthétique de la création verbale. Paris : Gallimard.

Barthes R. (1968). L'effet de réel. Communications, 11, 84-89.

Baudry, M., Bessonnat, D., Laparra, M. \& Tourigny, F. (1997). La maîtrise de la langue au collège. Paris : CNDP / Savoir-Livre.

Bautier, É. (2008). Ambitions et paradoxes des pratiques langagières scolaires : constructions au quotidien des inégalités sociales d'apprentissage. Actes du colloque "Ce que l'école fait aux individus ». Nantes: CENS et CREN, Université de Nantes. En ligne : http://www.cren-nantes.net/IMG/pdf/Bautier.pdf.

Bautier, É., Manesse, D., Peterfalvi, B. \& Vérin, A. (2000). Le cycle de vie du Cerisier: une narration "scientifique"? Repères, 21, 143-164.

Bernié, J.-P. (2002). L'approche des pratiques langagières scolaires à travers la notion de "communauté discursive": un apport à la didactique comparée ? Revue française de Pédagogie, 141, 77-88.

Britton, J. (1970). Language and Learning. Harmondsworth: Penguin.

Bronckart, J.-P. (1996). Activité langagière, textes et discours. Lausanne-Paris : Delachaux et Niestlé.

Combettes, B. \& Tomassone, R. (1988). Le texte informatif : aspects linguistiques. Bruxelles : De Boeck Université.

Crinon J., Marin B. \& Cautela A. (2008). Comprendre la révision collaborative : élaborer ou utiliser des critiques. Communication au Congrès mondial de linguistique française (CMLF 08), Paris, 9-12 juillet 2008. En ligne : $\mathrm{http}: / / \mathrm{www}$.linguistiquefrancaise.org.

Crinon J. \& Marin B. (2008). Apprendre à écrire des textes explicatifs en situation de révision collaborative. Communication au Colloque international «De la France au Québec, L’Écriture dans tous ses états », Poitiers, 12-15 novembre 2008. En ligne : http://www.poitou-charentes.iufm.fr/IMG/pdf/Crinon-Marin.pdf.

Crinon J. \& Marin B. (2010, soumis). Réviser à distance pour apprendre à écrire des textes narratifs. Revue française de Linguistique appliquée, $X V$.

Denhière, G. \& Baudet, S. (1992). Lecture, compréhension de texte et science cognitive. Paris : PUF.

Dolz, J. \& Schneuwly, B. (1998). Pour un enseignement de l'oral. Initiation aux genres formels à l'école. Issy les Moulineaux : ESF Éditeur.

Fabre, M. (1999). Situations-problèmes et savoir scolaire. Paris : PUF.

Garcia-Debanc, C. (1989). Le tri de textes, modes d'emploi. Pratiques, 62, 3-51. 
Groupe EVA (1996). De l'évaluation à la récriture. Paris : Hachette Education.

Jaubert, M. (2007). Langage et construction de connaissances à l'école. Un exemple en sciences. Pessac: Presses Universitaires de Bordeaux.

Kerbrat-Orecchioni, C. (2002). L'énonciation : De la subjectivité dans le langage. Paris : Armand Colin.

Kintsch, W. (1974). The Representation of Meaning in Memory. Hillsdale: Laurence Erlbaum Associates.

Labov, W. (1978). Le parler ordinaire. Paris : Les Éditions de Minuit (2 volumes).

Marin B. (2007). Culture écrite, culture orale : des ressources spécifiques. La Linguistique, 43(2),105-121.

Marin B. (2008). Jouer un rôle d'expert pour construire et mobiliser des ressources. Dilbilim, XVIII, 2-11.

McCutchen, D. (1986). Domain knowledge and linguistic knowledge in the development of writing ability. Journal of Memory and Language, 25, 431-444.

McNamara, D., Floyd, R., Best, R., \& Louwerse, M. (2004). World knowledge driving young readers' comprehension difficulties. In Y. B. Kafai, W. A. Sandoval, N. Enyedy, A. S. Nixon, \& F. Herrera (Eds). Proceedings of the sixth international conference of the learning sciences: Embracing diversity in the learning sciences (pp. 326-333). Mahwah: Laurence Erlbaum Associates.

Ohlsson, S. (2002). Generating and understanding qualitative explanations. In J. Otero, J.A. León, \& A.C. Graesser (Eds.), The Psychology of Science Text Comprehension (pp.91-128). Mahwah, NJ: Lawrence Erlbaum Associates.

Olson, D.R. (2008). History of schools and writing. In C. Bazerman (Ed.), Handbook of Research on Writing (pp. 283-292). New York, London: Lawrence Erlbaum Associates.

Rastier, F. (2007). Du réalisme au postulat référentiel. Revue Texto! En ligne: http://www.revuetexto.net/Inedits/Rastier/Rastier_Postulat.pdf.

Schneuwly, B et Dolz, J. (1997). Les genres scolaires. Des pratiques langagières aux objets d'enseignement. Repères, $15,27-40$.

Schneuwly, B. (Ed.) (1990). Diversifier l'enseignement du français écrit. Neuchâtel-Paris : Delachaux et Niestlé.

Tardy, C.M. \& Swales, J.M. (2008). Form, text organisation, genre, coherence and cohesion. In C. Bazerman (Ed.), Handbook of Research on Writing (pp. 565-581). New York, London: Laurence Erlbaum Associates.

Vergnaud, G. (2003). La conception clef de voûte du rapport entre pratique et théorie. In Analyse de pratiques et professionnalité des enseignants. Actes de la DESCO (pp. 48-55). Versailles: CRDP de l'académie de Versailles.

Wenger, E. (1998). Communities of Practises. Cambridge: Cambridge University Press.

\footnotetext{
${ }^{1}$ C'est l'opposition fondamentale, selon Bronckart (1996), entre l'« exposer » et le « raconter », interprétés ou non « à l'aune des critères de validité du monde ordinaire » (p. 156).

${ }^{2}$ Chacune des quatre séquences échelonnées du mois de novembre au mois de mai d'une année scolaire comporte quatre séances de travail qui seront détaillées plus loin.

${ }^{3}$ Nous avons aussi procédé à d'autres analyses des corpus ainsi constitués, pour étudier l'évolution des compétences en écriture des élèves récepteurs de critiques de textes de fiction (Marin, 2008), de textes explicatifs (Marin, 2007) et la comparaison des compétences d'écriture acquises par les élèves émetteurs et récepteurs de critiques de textes explicatifs (Crinon, Marin \& Cautela, 2008 ; Crinon \& Marin, 2008).

${ }^{4}$ De 2006 à 2009.

${ }^{5}$ Les niveaux des élèves ont été établis à l'aide de tests de lecture (remise en ordre et importance relative de l'information).

${ }^{6}$ Nous disposons ainsi pour chacun de ces 72 élèves, de ses quatre textes en version 1 et en version 2, soit 576 textes et de quatre séries de critiques adressées aux correspondants de son groupe.
} 
Didactique et enseignement, français langue maternelle, français langue seconde DOI $10.1051 / \mathrm{cmlf} / 2010207$

\footnotetext{
${ }^{7}$ Pour les trois premières séquences : Victor Astafiev, Perdu dans la tä̈ga, Flammarion Castor Poche. Pour la quatrième séquence : Jean Ollivier, Le cri du Kookabura, Casterman.

${ }^{8}$ On sait, depuis notamment les travaux de McCutchen (1986), l'importance tout à la fois des connaissances sur le domaine et des connaissances linguistiques dans le développement des compétences d'écriture des élèves de l'école élémentaire.

${ }^{9}$ Est considérée comme pertinente toute information recevable sur les plans sémantique et logique.

${ }^{10}$ L'orthographe des textes d'élèves a été normalisée.

${ }^{11}$ Entre la version 1 et la version 2, les ajouts sont mis en gras, les suppressions entre parenthèses, les remplacements en italiques et les déplacements entre crochets.

${ }^{12}$ En dépit des maladresses linguistiques liées à une scolarisation tardive de cette élève en milieu francophone.

${ }^{13}$ Ce texte, de ce point de vue, est marginal dans notre corpus à ce moment de l'année. Il n'est pas indifférent de noter qu'Élodie était absente lors de l'écriture de la version 2 de la séquence précédente et n'a pu bénéficier du travail d'élaboration afférent.
} 